\title{
Eficacia de L-carnitina vía oral en el incremento ponderal en recién nacidos prematuros. Ensayo clínico controlado aleatorizado doble ciego
}

\author{
Efficacy of oral L-carnitine in weight gain in preterm newborns. \\ Double-blind, randomized controlled trial \\ Gabriela Virginia Escudero-Lourdes, * Francisco Javier Figueroa-Vallejo, ${ }^{\ddagger}+$ \\ Peter Bisset-Mandeville, ${ }^{\S}$ Manuel Rodríguez-Martínez, ${ }^{\Uparrow}$ Francisco Hernández-Sierra॥ \\ * Coordinación Delegacional de Educación en Salud, IMSS; ‡ Facultad de Medicina de la Universidad Autónoma del \\ Estado de Morelos; ${ }^{\S}$ Departamento de Epidemiología y Estadística de la Escuela de Medicina de la Universidad de San \\ Luis Potosí; " Departamento de Fisiología de la Escuela de Medicina de la Universidad de San Luis Potosí; "Departamento \\ de Epidemiología de la Escuela de Medicina de la Universidad de San Luis Potosí. San Luis Potosí, México.
}

\begin{abstract}
RESUMEN
Introducción: En México se estima que entre ocho y 10\% de los nacimientos son recién nacidos pretérmino (RNPT). Lograr un adecuado crecimiento y desarrollo son de los retos en la atención de los RNPT, por lo que se recomienda suplementación de nutrientes, como levocarnitina. Objetivo: Evaluar la eficacia de levocarnitina oral en el incremento ponderal en RNPT comparado con placebo. Material y métodos: Se realizó un ensayo clínico aleatorizado, doble ciego, comparado con placebo en un Hospital General de Zona, de San Luis Potosí, México. Se incluyeron RNPT con peso menor a $1.8 \mathrm{~kg}$, en condiciones para recibir alimentación enteral. El grupo experimental recibió $100 \mathrm{mg} / \mathrm{kg} / \mathrm{día}$ de levocarnitina y el grupo control solución glucosada al $5 \%$. Se evaluó la ganancia de peso en gramos a los siete, 14 y 21 días, así como el tiempo de estancia hospitalaria. El análisis estadístico se realizó con Chi-cuadrada y $t$ de Student. Resultados: Fueron 32 RNPT en el grupo de levocarnitina y 28 del grupo placebo. Al analizar el peso, el grupo experimental tuvo ganancia de peso en promedio de $167.8 \mathrm{~g} \pm 54.97,203.5 \mathrm{~g} \pm 65.05$ y $202.1 \mathrm{~g} \pm 57.58$, en las tres mediciones semanales. Lo cual fue mayor $(p<0.05)$ que
\end{abstract}

\begin{abstract}
Introduction: In Mexico, it is estimated that eight to $10 \%$ of births are preterm newborns. Achieving adequate growth and development are one of the challenges of caring for prematures, which is why supplementation of nutrients, such as levocarnitine, is recommended. Objective: To assess the efficacy of oral levocarnitine in weight gain of prematures, compared with placebo. Material and methods: A randomized, doubleblind controlled trial, compared with placebo, was carried out in a General Hospital, San Luis Potosí, Mexico. Patients weighing less than $1.8 \mathrm{~kg}$ were included, who had conditions to receive enteral feeding. The experimental group received $100 \mathrm{mg} / \mathrm{kg} /$ day of levocarnitine, and the control group 5\% glucose solution. Weight gain (grams) at 7, 14 and 21 days, as well as length of hospital stay, were evaluated. Statistical analysis was performed with Chi-square and Student's $t$. Results: There were 32 prematures in the levocarnitine group and 28 in the placebo group. When analyzing the weight, the experimental group had an average weight gain of $167.8 \mathrm{~g}$ $\pm 54.97,203.5 \mathrm{~g} \pm 65.05$ and $202.1 \mathrm{~g} \pm 57.58$, in the three weekly measurements. Which was higher $(p<0.05)$ than in the control group: $143.4 \mathrm{~g} \pm 60.32,174.5 \mathrm{~g} \pm 52.86$ and 151
\end{abstract}

+ Correspondencia: FJFV, fanjav@hotmail.com

Conflicto de intereses: Los autores declaran que no tienen.

Citar como: Escudero-Lourdes GV, Figueroa-Vallejo FJ, Bisset-Mandeville P, Rodríguez-Martínez M, Hernández-Sierra F. Eficacia de Lcarnitina vía oral en el incremento ponderal en recién nacidos prematuros. Ensayo clínico controlado aleatorizado doble ciego. Rev Mex Pediatr. 2020; 87(5): 183-188. https://dx.doi.org/10.35366/97172 
en el grupo control: $143.4 \mathrm{~g} \pm 60.32,174.5 \mathrm{~g} \pm 52.86$ y 151 $\mathrm{g} \pm 44.58$, respectivamente. Además, el grupo experimental tuvo menor número de días de estancia hospitalaria, $15.5 \pm$ 7.1 vs $18.1 \pm 8.6$. Conclusión: En RNPT sin complicaciones, la administración de levocarnitina oral es eficaz para mejorar la ganancia de peso y, muy probablemente, para reducir la estancia intrahospitalaria.

Palabras clave: Levocarnitina, recién nacidos, prematuro, ganancia de peso, estancia intrahospitalaria. $g \pm 44.58$, respectively. In addition, the experimental group had fewer days of hospitalization, $15.5 \pm 7.1$ vs $18.1 \pm 8.6$. Conclusion: In preterm newborns without complications, the administration of oral levocarnitine is effective in improving weight gain and probably in reducing hospital stay.

Keywords: Levocarnitine, preterm, newborn, weight gain, hospital stay.

\section{INTRODUCCIÓN}

La Organización Mundial de la Salud (OMS) define como nacimiento pretérmino al que se produce antes de las 37 semanas de gestación (SDG). ${ }^{1,2}$ En México se estima que de ocho a $10 \%$ de los nacimientos son recién nacidos pretérmino (RNPT), con un aumento en la incidencia de prematuros de entre 28-32 SDG. ${ }^{3}$ En el año 2005, en el Hospital General de Zona No. 1 de San Luis Potosí del Instituto Mexicano del Seguro Social (IMSS) la incidencia de RNPT fue de 81:1,000 recién nacidos. ${ }^{4}$

En la actualidad, sobreviven más de 90\% de los RNPT mayores de 30 semanas de gestación; ${ }^{5}$ pero la mayoría requiere atención médica especializada. ${ }^{6,7}$ Uno de los problemas es el relacionado con el crecimiento, lo cual se vuelve un reto, por lo que en muchos casos se requiere la suplementación especial de nutrientes, como es el caso de la levocarnitina. ${ }^{8-10}$

La levocarnitina es un isómero activo y levógiro de carnitina, derivado de la lisina y metionina, con fórmula química de ácido 3-hidroxi-4-N-trimetil aminobutírico, siendo un compuesto identificado por primera vez en el músculo esquelético. ${ }^{11,12}$ La levocarnitina tiene dos funciones principales: la primera es facilitar el ingreso mitocondrial de ácidos grasos de cadena larga para su beta-oxidación y formación de adenosina trifosfato (ATP), sustrato importante para la obtención de energía. La segunda función es remover ésteres de acil-CoA, ayudando a la mitocondria a recuperar coenzima A libre. ${ }^{13,14}$

La levocarnitina exógena se absorbe principalmente en el yeyuno, ingresa a la circulación y se distribuye ampliamente en el organismo, con un pico máximo a las 3.3 horas de su ingesta y una biodisponibilidad de 15 a $16 \%$. Se excreta a las 24 horas por vía urinaria en $83.5 \%$. Carece de efectos tóxicos, incluso a dosis de $15 \mathrm{~g}$ diarios, aunque dosis mayores a $3 \mathrm{~g}$ se asocian con reblandecimiento de heces y sudoración con olor a pescado (incidencia $<2 \%$ ), lo cual se evita al reducir la dosis. La dosis recomendada en niños con deficiencias primarias es $100 \mathrm{mg} / \mathrm{kg}$ de peso por día, dividida en dos a tres dosis. ${ }^{12}$ No se han reportado interacciones medicamentosas. ${ }^{15}$ Las indicaciones actuales de uso de levocarnitina son: deficiencias primarias de carnitina, alto consumo de carnitina, pérdida de carnitina, alteraciones metabólicas y endocrinas, así como falta de síntesis, como es el caso de los RNPT. ${ }^{12}$

En recién nacidos $(\mathrm{RN})$, la síntesis de levocarnitina es limitada porque el metabolismo es inmaduro. ${ }^{16} \mathrm{~A}$ lo anterior, se debe agregar que en los RNPT la reserva de carnitina es $60 \%$ menor que en los de término, por lo que sus requerimientos son mayores para su óptimo crecimiento. ${ }^{8-10,17,18}$

Con estos antecedentes, el objetivo del estudio fue evaluar la eficacia de la administración de levocarnitina por vía oral para el incremento ponderal de RNPT comparado con placebo.

\section{MATERIAL Y MÉTODOS}

Se realizó un ensayo clínico doble ciego, paralelo, con asignación aleatoria, comparado con placebo, para evaluar el efecto sobre el incremento de peso de RNPT con levocarnitina vía enteral a dosis de $100 \mathrm{mg} / \mathrm{kg} /$ día. El grupo control recibió solución glucosada al 5\%.

Los criterios de selección fueron: RN de 28 a 33 SDG según la escala de Capurro, que ingresaron a la sala de incremento ponderal del servicio de Neonatología en el Hospital General de Zona No. 1 del IMSS, en la ciudad de San Luis Potosí, México. Además, deberían tener peso adecuado para la edad gestacional, sin complicaciones, que tolerarán la alimentación por sonda nasogástrica, recibiendo al menos $100 \mathrm{~mL}$ de leche materna y/o fórmula en 24 horas, y con autorización por los padres o responsables del menor mediante consentimiento informado.

Se excluyeron pacientes con malformaciones congénitas mayores, enfermedades metabólicas (por 
ejemplo, hipotiroidismo congénito, hiperplasia suprarrenal congénita, hipoglucemia persistente, etcétera), o aquéllos con antecedente materno de diabetes mellitus o hipotiroidismo, o bien, que recibieran medicamentos que disminuyen la producción endógena de levocarnitina como ácido valproico o análogos nucleósidos. Por último, fueron eliminados quienes fueron trasladados a otro hospital o que fueron egresados antes de concluir el estudio.

Se calculó el tamaño de muestra bajo el supuesto de que la levocarnitina fuera superior al placebo en $25 \%$ para el incremento de peso. Tomando en cuenta alfa de $95 \%$ y un poder de $80 \%$ se estimó que eran requeridos 30 pacientes por grupo.

Las variables de desenlace principales fueron: 1) aumento de peso (en gramos) acumulado semanalmente hasta lograr un peso de $1.8 \mathrm{~kg}$ o los 21 días de iniciado el estudio, y 2) días de estancia.

La asignación aleatoria fue realizada en un programa estadístico, y por técnica de aleatorización simple se establecieron los códigos de tratamiento por grupo. Para conservar el doble ciego del estudio, el producto de investigación y la solución con placebo fueron contenidos en frascos con las mismas características físicas (tamaño y color), marcados con letras A y B para diferenciar los grupos, los cuales se prepararon con técnica estéril. La posología usada fue de $100 \mathrm{mg} / \mathrm{kg}$ de peso de levocarnitina o su equivalente en mililitros de solución glucosada al 5\%. Para asegurar el depósito de ambos en cavidad gástrica, se administraron por sonda orogástrica, posterior instilación de 1 a $2 \mathrm{~mL}$ de agua bidestilada. Una vez iniciada la administración de activo o placebo se continuó la periodicidad hasta el egreso de cada paciente.

Las incubadoras y los expedientes de los RNPT se marcaron con las letras A o B, según el grupo al que correspondieron. Para las incubadoras se registró cada ocho horas la temperatura, a fin de mantener la eutermia de los RNPT.

Se capacitó al personal de enfermería de los turnos matutino y fines de semana para realizar la medición de peso de manera adecuada, con una consistencia intraobservador del 0.99 . El peso se tomó con una báscula fija calibrada, de manera diaria a las 08:30, con los RNPT en ayuno, sin ropa y sin pañal. La báscula era electrónica marca SECA, la cual se verificó su calibración antes del inicio del estudio.

La cantidad de leche materna y fórmula ingerida por paciente se sumó y registró por paciente al final del día en la hoja de enfermería y en la hoja de recolección de datos asignada para el estudio. Los incre- mentos en mililitros de leche materna o fórmula se realizaron de acuerdo con la tolerancia del paciente, en un rango de 15 a $25 \mathrm{~mL}$, según lo indicado por el médico tratante.

Respecto a datos bioquímicos, se midió urea y hemoglobina de manera semanal.

\section{Análisis estadístico}

Los datos cualitativos se presentan como frecuencias y porcentajes, mientras que los cuantitativos con promedio y desviación típica (DT), dado que mostraron distribución normal con el método de Shapiro Wilks. La comparación entre grupos fue con prueba de Chicuadrada y con t de Student. El nivel de significancia estadística se estableció con una $\mathrm{p} \leq 0.05$.

\section{Consideraciones éticas}

El estudio fue aprobado por el Comité de Investigación y por el Subcomité de Bioética del mismo hospital. Los padres o tutores de los RNPT fueron informados acerca de los procedimientos a realizar y dieron su consentimiento informado para la participación de los RNPT en el estudio.

\section{RESULTADOS}

El tamaño muestral requerido se completó entre enero de 2006 y febrero de 2007. Una vez aleatorizados, 32 RNPT recibieron levocarnitina y 28 solución glucosada al $5 \%$. Ningún paciente fue eliminado del estudio y el código de cada paciente se mantuvo cegado hasta que se concluyó el análisis de los datos (Figura 1).

Las variables demográficas basales para ambos grupos se presentan en la Tabla 1; como se muestra, no se encontraron diferencias estadísticamente significativas entre los grupos ( $p>0.05)$.

En los dos grupos, el peso mostró un patrón uniforme hacia el incremento por día. Sin embargo, fue mayor en el grupo que recibió levocarnitina (104.5 g) con una ganancia ponderal total en promedio de 573.4 g, en comparación a 468.9 g del grupo placebo (Figura 2). Asimismo, el incremento de peso promedio diario fue mayor en el grupo de levocarnitina que el del grupo control, $27.29 \mathrm{~g}$ vs $4.97 \mathrm{~g}$.

$\mathrm{Al}$ analizar el peso de manera semanal, el grupo de levocarnitina tuvo ganancia de peso en promedio de $167.8 \mathrm{~g}(\mathrm{DT} \pm 54.97), 203.5 \mathrm{~g}(\mathrm{DT} \pm 65.05)$ y $202.1 \mathrm{~g}$ 
(DT \pm 57.58 ), a la primera, segunda y tercera semana, respectivamente. Los cuales fueron mayores que lo registrado en el grupo control: $143.4 \mathrm{~g}$ (DT \pm 60.32$), 174.5$ $\mathrm{g}(\mathrm{DT} \pm 52.86)$ y $151 \mathrm{~g}(\mathrm{DT} \pm 44.58)$, respectivamente.

Por último, al comparar el tiempo de estancia el grupo de levocarnitina tuvo menor días de hospitalización (15.5 \pm 7.1 vs $18.1 \pm 8.6)$, pero sin llegar a ser estadísticamente significativo.

\section{DISCUSIÓN}

Para que un RNPT pueda egresarse del hospital, el incremento ponderal es uno de los principales objetivos, lo cual puede representar un reto en el cuidado médico. Para mejorar el crecimiento intrahospitalario del RNPT, se requiere suplementar de nutrientes para lograr el incremento de peso deseado, ${ }^{19-23}$ dentro de éstos se encuentra la levocarnitina, que ayuda tanto en la tolerancia de la nutrición parenteral ${ }^{24-26}$ como a mejorar niveles de levocarnitina, que generalmente están disminuidas en los prematuros. ${ }^{17-18}$

Este estudio se realizó para evaluar si la suplementación de levocarnitina oral a dosis de $100 \mathrm{mg} / \mathrm{kg}$ al día, vía enteral, mejora la ganancia de peso del RNPT, y su posible impacto en la estancia hospitalaria, al ser comparado con placebo, haciendo mención que, en el momento del desarrollo del estudio, no existían datos en prematuros mexicanos. Los resultados comprobaron que la administración de levocarnitina es efectiva para incrementar el peso del RNPT, sin mostrar efectos adversos (como diarrea u olor a pescado), lo cual es similar a lo demostrado por otros autores. ${ }^{24-26}$ Además, se pudo observar que el uso de levocarnitina redujo el tiempo de hospitalización, lo cual no ha sido reportado previamente. Lo anterior, debido a que los RNPT alcanzaron más rápidamente la meta de peso necesario para ser egresados.

Si bien, el diseño del estudio (ensayo clínico aleatorizado y doble ciego) permite determinar la validez de nuestros resultados, puesto que constituyen el más alto grado de evidencia, también es importante considerar las limitantes, una de las cuales es no haber evaluado los niveles séricos de levocarnitina, ya que éstos hubieran sido de utilidad para conocer si había deficiencias entre los grupos al inicio del estudio y su comportamiento a lo largo de éste. Sin embargo, puede

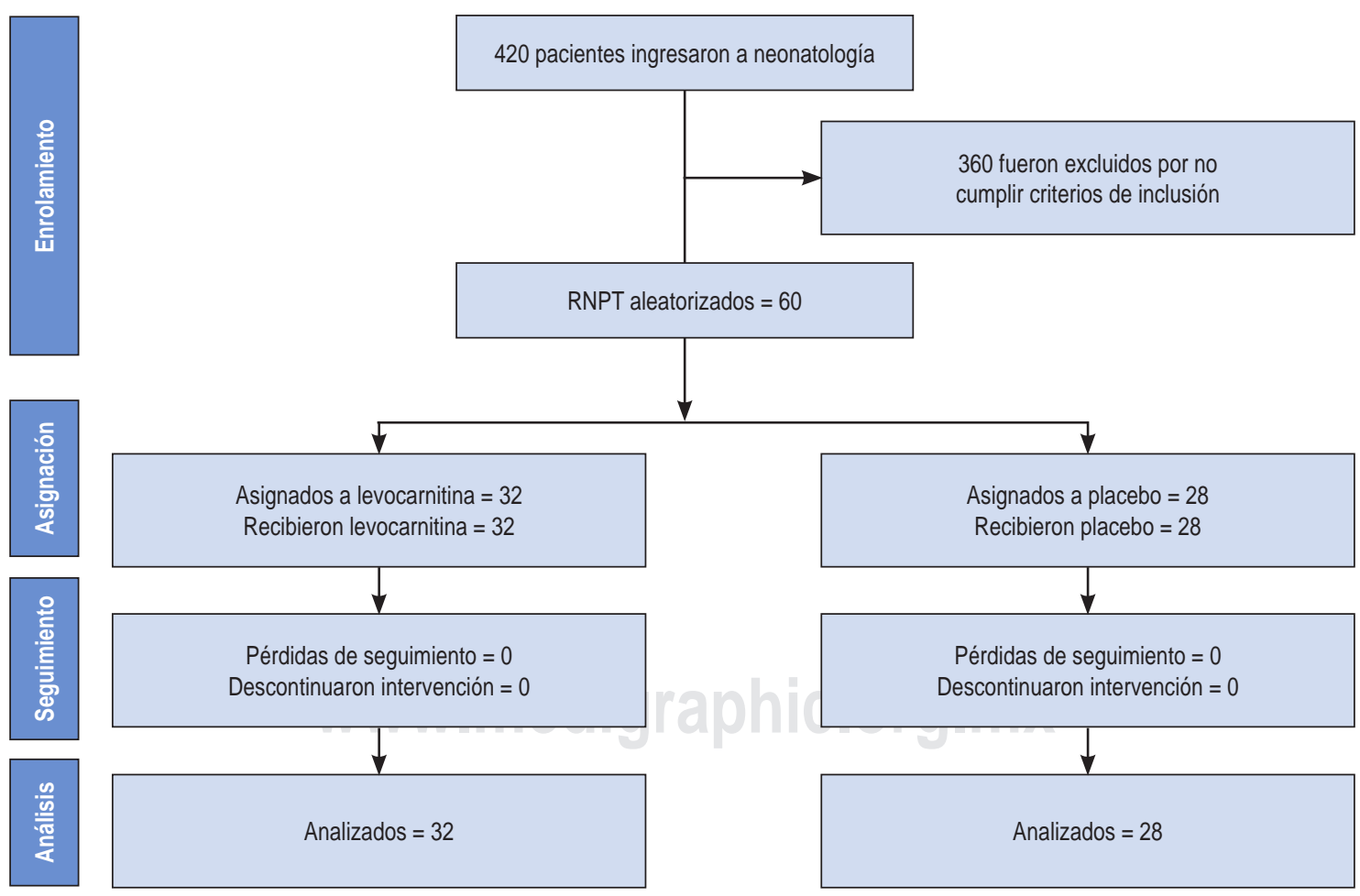

Figura 1: Diagrama de CONSORT del estudio.

RNPT=recién nacidos pretérmino. 
Tabla 1: Comparación de las características al inicio de la intervención entre los grupos de estudio.

\begin{tabular}{|c|c|c|c|}
\hline Característica & $\begin{array}{c}\text { Grupo levocarnitina } \\
n=32 \\
\text { promedio } \pm \text { DT }\end{array}$ & $\begin{array}{c}\text { Grupo placebo } \\
n=28 \\
\text { promedio } \pm \text { DT }\end{array}$ & $p$ \\
\hline Peso al nacimiento $(\mathrm{g})$ & $1,368.6 \pm 381.5$ & $1,396.0 \pm 384.2$ & 0.86 \\
\hline Peso al ingreso del estudio ( $\mathrm{g}$ ) & $1,290.3 \pm 244.4$ & $1,246.7 \pm 219.6$ & 0.32 \\
\hline SDG & $30.9 \pm 1.5$ & $31 \pm 1.9$ & 0.89 \\
\hline VEU (días) & $21.6 \pm 4.1$ & $19.2 \pm 3.4$ & 0.46 \\
\hline \multicolumn{4}{|l|}{ Sexo, n (\%) } \\
\hline Femenino & $18(56.2)$ & $14(50)$ & 0.82 \\
\hline Masculino & $14(43.8)$ & $14(50)$ & \\
\hline Leche materna basal $(\mathrm{mL})$ & $79.0 \pm 72.6$ & $81.8 \pm 79.8$ & 0.32 \\
\hline $\mathrm{Hb}(\mathrm{mg} / \mathrm{dL})$ & $11.8 \pm 2.6$ & $12.4 \pm 2.3$ & 0.46 \\
\hline Urea (mg/dL) & $22.1 \pm 14.3$ & $19.7 \pm 15.1$ & 0.50 \\
\hline
\end{tabular}

SDG = semanas de gestación; VEU = días de vida extrauterina; $\mathrm{Hb}$ = hemoglobina; $\mathrm{DT}$ = desviación típica.

ser difícil atribuir que los niveles de levocarnitina sean sólo debido a la suplementación, ya que en el prematuro existe producción endógena. Otra posible limitante es no haber incluido prematuros complicados, por lo cual los resultados no se pueden extrapolar a ese grupo. Asimismo, este estudio sólo se desarrolló en un centro hospitalario, por lo que en otras unidades hospitalarias los resultados pudieran no ser reproducibles; de ahí que parece conveniente en el futuro la realización de estudios multicéntricos.

Por último, dado que se ha descrito que niveles de levocarnitina adecuados pueden tener alguna acción a nivel cerebral, ${ }^{9}$ en el futuro sería interesante evaluar el

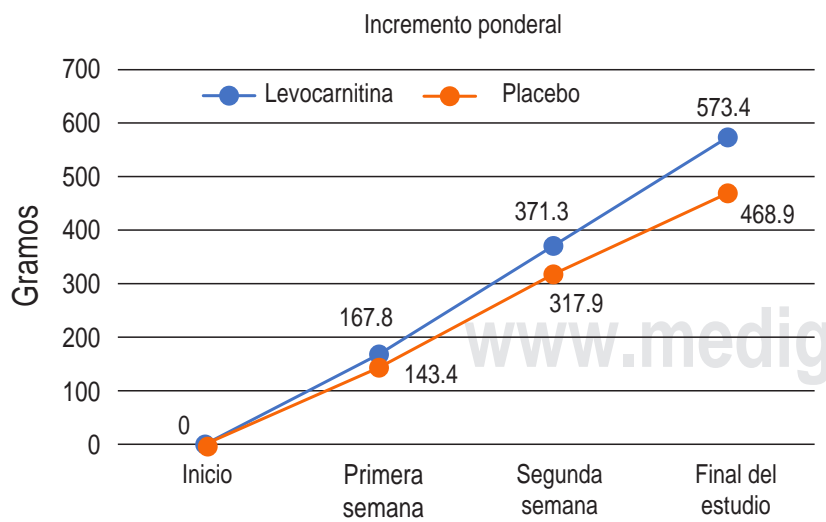

Figura 2: Incremento de peso semanal en gramos por grupo de estudio. neurodesarrollo en los primeros años de vida en RNPT que se suplementan con levocarnitina.

\section{CONCLUSIÓN}

En RNPT la administración de $100 \mathrm{mg} / \mathrm{kg} /$ día de levocarnitina por vía enteral mejora la ganancia de peso y, probablemente, los días de estancia intrahospitalaria.

\section{REFERENCIAS}

1. Organización Mundial de la Salud [Internet]. Nacimientos prematuros. Suiza: OMS; c2019. 19 febrero 2019 [citado 18 jun 2019]. Disponible en: https://www.who.int/es/news-room/factsheets/detail/preterm-birth.

2. Gómez-Gómez M, Danglot-Banck C, Aceves-Gómez M. Clasificación de los niños recién nacidos. Rev Mex Pediatr. 2012; 79(1): 32-39.

3. Villanueva LA, Contreras AK, Pichardo M, Rosales J. Perfil epidemiológico del parto prematuro. Ginecol Obstet Mex. 2008; 76(9): 542-548.

4. IMSS. Sistema de información de morbilidad. 2006. Delegación San Luis Potosí S.L.P. H.G.Z. 1.

5. Goldenberg RL. The management of preterm labor. Obstet Gynecol. 2002; 100(5Pt1): 1020-1037.

6. Martínez y Martínez R. La salud del niño y el adolescente. 8a ed. México: Manual Moderno; 2017.

7. Mendoza LA, Claros DI, Mendoza LI, Arias MD, Peñaranda CB. Epidemiología de la prematuridad, sus determinantes y prevención del parto prematuro. Rev Chil Obstet Ginecol. 2016; 81(4): 330-342.

8. Clark MA, Stein REK, Silver EJ, Khalid S, Fuloria M, EstebanCruciani NV. Carnitine deficiency in preterm infants: A national survey of knowledge and practices. J Neonatal Perinatal Med. 2017; 10(4): 381-386. 
9. Baronio F, Righi B, Righetti F, Bettocchi I, Ortolano R, Faldella $G$ et al. Carnitine longitudinal pattern in preterm infants $<1800 \mathrm{~g}$ body weight: a case-control study. Pediatr Res. 2019; 86(5): 646-650.

10. Liu Q, Wu J, Shen W, Wei R, Jiang J, Liang J et al. Analysis of amino acids and acyl carnitine profiles in low birth weight, preterm, and small for gestational age neonates. J Matern Fetal Neonatal Med. 2017; 30(22): 2697-2704.

11. Vacha GM, Giorcelli G, Siliprandi N, Corsi M. Favorable effects of L-carnitine treatment on hypertriglyceridemia in hemodialysis patients: decisive role of low levels of high-density lipoproteincholesterol. Am J Clin Nutr. 1983; 38(4): 532-540.

12. Laboratorios Grossman. Información para prescribir Cardispán ${ }^{\circledR}$ Solución Pediátrica al 10\% y Solución Oral al 30\%. 2016. México.

13. Rebouche CJ, Engel AG. Tissue distribution of carnitine biosynthetic enzymes in man. Biochim Pharmacol. 1987; 36(20): 3045-3049.

14. Almannai M, Alfadhel M, El-Hattab AW. Carnitine inborn errors of metabolism. Molecules. 2019; 24(18): 3251.

15. Rebouche CJ. Carnitine. In: Shils ME, Olson JA, Shike M, Ross AC, editors. Modern nutrition in health and disease. 9th ed. Baltimore: Lippincott Williams \& Wilkins; 1999. pp. 505-512.

16. Montgomery R, Espector A. Bioquímica casos y texto. 6a ed. España: Elsevier; 1999.

17. Schiff D, Chan G, Seccombe D, Hahn P. Plasma carnitine levels during intravenous feeding of the neonate. J Pediatr. 1979; 95(6): 1043-1046.
18. Sánchez-Pintos P, Pérez-Muñuzuri A, Cocho JÁ, FernándezLorenzo JR, Fraga JM, Couce ML. Evaluation of carnitine deficit in very low birth weight preterm newborns small for their gestational age. J Matern Fetal Neonatal Med. 2016; 29(6): 933-937.

19. Engel AG, Rebouche CJ. Carnitine metabolism and inborn errors. $J$ Inherit Metab Dis. 1984; 7 Suppl 1: 38-43.

20. Schmidt-Sommerfeld E, Penn D, Wolf H. Carnitine blood concentrations and fat utilization in parenterally alimented premature newborn infants. J Pediatr. 1982; 100(2): 260-264.

21. Mitchell ME, Synder EA. Dietary carnitine effects on carnitine concentrations in urine and milk in lacting women. Am J Clin Nutr. 1991; 54(5): 814-820.

22. Usher R, McLean F. Intrauterine growth of live-born Caucasian infants at sea level: standards obtained from measurements in 7 dimensions of infants born between 25 and 44 weeks of gestation. $J$ Pediatr. 1969; 74(6): 901-910.

23. MacDonald MG, Fletcher MA, Avery GB. Neonatología, fisiopatología y manejo del recién nacido. $5^{\underline{a}}$ ed. Argentina: Editorial Médica Panamericana S.A.; 2001.

24. Bonner CM, DeBrie KL, Hug G, Landrigan E, Taylor BJ. Effects of parenteral L-carnitine supplementation on fat metabolism and nutrition in premature neonates. J Pediatr. 1995; 126(2): 287-292.

25. Coran AG, Drongowski R, Baker PJ. The metabolic effects of oral L-carnitine administration in infants receiving total parenteral nutrition with fat. J Pediatr Surg. 1985; 20(6): 758-764.

26. Curran JS, Williams PR, Kanarek KS, Novak M, Monkus EF. An evaluation of orally supplemented L-carnitine in premature infants receiving Intralipid 20\%. Acta Chir Scand Suppl. 1983; 517: 157-164. 